\title{
The minimax risk of truncated series estimators for symmetric convex polytopes
}

\author{
Adel Javanmard* $\quad$ Li Zhang ${ }^{\dagger}$
}

August 22, 2021

\begin{abstract}
We study the optimality of the minimax risk of truncated series estimators for symmetric convex polytopes. We show that the optimal truncated series estimator is within $O(\log m)$ factor of the optimal if the polytope is defined by $m$ hyperplanes. This represents the first such bounds towards general convex bodies. In proving our result, we first define a geometric quantity, called the approximation radius, for lower bounding the minimax risk. We then derive our bounds by establishing a connection between the approximation radius and the Kolmogorov width, the quantity that provides upper bounds for the truncated series estimator. Besides, our proof contains several ingredients which might be of independent interest: 1 . The notion of approximation radius depends on the volume of the body. It is an intuitive notion and is flexible to yield strong minimax lower bounds; 2. The connection between the approximation radius and the Kolmogorov width is a consequence of a novel duality relationship on the Kolmogorov width, developed by utilizing some deep results from convex geometry [1, 19, 8, .
\end{abstract}

\section{Introduction}

In this paper, we study the minimax risk of estimators for symmetric convex polytopes. We show that for a symmetric convex polytope defined by $m$ hyperplanes, the truncated series estimator, a special type of linear estimator, is within $O(\log m)$ factor of the optimal.

In non-parametric statistics, the minimax risk of an estimator measures the worst case expected loss of the estimator for input coming from some subset $X \subseteq \mathbb{R}^{n}$ (see Section 2.2 for a formal definition). Tremendous work has been done on understanding the optimal minimax risk for various families of $X$. But it is usually very difficult to design the optimal estimator. The truncated series estimator is a family of linear estimator that simply projects an observation to a properly chosen subspace. Despite its simplicity, the truncated series estimator is surprisingly powerful and is shown to be nearly optimal for wide families of convex bodies. [17] shows that such estimator is nearly optimal for ellipsoids. In [5], it is shown that it is nearly optimal for the wider family of orthosymmetric and quadratically convex objects, including $\ell_{p}$ balls for $p \geq 2$.

In this paper, we show that the power of truncated series estimator extends to the rich class of symmetric polytopes. Specifically, we show that for a symmetric convex polytope

\footnotetext{
*Department of Electrical Engineering, Stanford University, CA. The work was partially done during an internship at Microsoft Research.

${ }^{\dagger}$ Microsoft Research Silicon Valley, Mountain View, CA
} 
defined by $m$ hyperplanes, the truncated series estimator is within $O(\log m)$ factor of the optimal. Previously, such results have only been obtained for particular family of convex polytopes, such those corresponding to the Lipschitz condition [14] or satisfying certain isometric conditions [18. As a motivating example, we discuss one application of our result in estimating values of a Lipschitz function.

Example. One important estimation problem in the literature is the estimation of functions satisfying certain continuity or Lipschitz conditions from noisy measurements. Consider a univariate Lipschitz function $f:[0,1] \rightarrow \mathbb{R}$. Suppose that $x_{i}=f\left(t_{i}\right)$ for $i=1, \ldots, n$, and we have measurements $y_{i}$ according to the model $y_{i}=x_{i}+w_{i}$ for some gaussian noise $w_{i}$. Then Lipschitz condition, with constant $L$, translates to the linear constraints:

$$
\left|x_{i+1}-x_{i}\right| \leq L\left|t_{i+1}-t_{i}\right|, \text { for } i=1, \ldots, n-1 \text {. }
$$

Now, we are interested in estimating $x_{i}$ from $y_{i}$. A key observation is that the vector $x=\left(x_{1}, \cdots, x_{n}\right)$ falls in the set $X$, where

$$
X=\left\{x:\left|x_{i+1}-x_{i}\right| \leq L\left|t_{i+1}-t_{i}\right|, \text { for } 1 \leq i \leq n-1\right\} .
$$

Note that $X$ is a symmetric convex polytope.

When the sampling is uniform, i.e. $t_{i}=(i-1) /(n-1)$, then $X$ has a more special form of $X=\left\{x:\left|x_{i+1}-x_{i}\right| \leq L /(n-1)\right\}$. In this case, previous work [14, 20] has shown that the best truncated series estimator is nearly optimal. As a consequence of our work, the truncated series estimator is nearly optimal (within $O(\log n)$ factor) for estimating Lipschitz function at arbitrary sample set $\left\{t_{1}, \ldots, t_{n}\right\}$.

At the high level, the proof of our results follows a very simple strategy. We choose a family of "obstruction objects" for which we can obtain lower bounds of the minimax risk. Then we show a "duality" result that if $X$ does not have a good truncated series estimator, then it will have to contain a "large" obstruction, and therefore no estimator can do well on $X$. Of course, the difficulty is in choosing the obstruction so that we can prove the corresponding duality result. Some natural obstructions include hyper-rectangles and Euclidean balls, for which we know very tight minimax lower bound. But they turn out to be too restrictive to allow a strong enough duality result. To overcome this difficulty, we consider a broader family consisting of objects which contain a "non-negligible" fraction of a "large" Euclidean ball; whence we are able to establish a desired duality relationship.

More specifically, we first define a geometric measure for any set, called approximation radius, and then develop a lower bound technique which bounds the minimax risk of any body by its approximation radius. Intuitively, the approximation radius of an object $X$ is the maximum radius of a ball with "non-negligible" volume fraction inside $X$. By refining the technique in [23], we can show that the minimax risk of $X$ is asymptotically as large as that of the ball with $X$ 's approximation radius (see Theorem 3.2). On the other hand, the minimax risk of truncated series estimator is determined by the Kolmogorov width of the object. Our bound is then derived by establishing a connection between the Kolmogorov widths and the approximation radius of the symmetric convex bodies (see Theorem 3.4). For the connection, we first derive a duality relationship between the Kolmogorov widths of $X$ and its polar dual $X^{\circ}$ (see Theorem 3.3), by utilizing some results from convex geometry started in [1]. The Kolmogorov width of $X^{\circ}$ is then shown, by probabilistic arguments, to be intimately related to the approximation radius of $X$. 


\section{$1.1 \quad$ Related work}

There is a vast body of work on the minimax estimators and it is beyond the scope of this paper to survey all of them. We refer to [14, 20, 11] for comprehensive survey and will describe some work most relevant to this paper. Since we focus on the mean squared error (MSE), all the subsequent discussion is in the context of MSE.

The minimax bounds have been developed for various families of convex bodies through intensive research in the past decades. Asymptotically tight bounds have been proposed for convex bodies that correspond to various continuity or energy conditions; the classes of Hölder balls, Sobolev balls, and Besov balls. We refer to Chapter 2.8 in [20] for a comprehensive recount of the references. Despite these remarkable results, it is still largely unknown how to compute the minimax risk for an arbitrary convex body. Some previous work does attempt to deal with less specific objects (see [18] and the references therein), but all the optimality results are under (fairly strong) isometric assumption about the objects.

On the other hand, the truncated series estimator has a nice geometric interpretation and is related to the classical Kolmogorov width of the underlying space. In addition to its simplicity, [5] shows that it is asymptotically optimal for the classes of orthosymmetric and quadratically convex objects. This includes the class of diagonally stretched $\ell_{p}$ balls for $p \geq 2$. Present paper shows that the power of truncated series estimators also extend to the family of symmetric convex polytopes, as long as the polytope is defined by $n^{O(1)}$ hyperplanes.

To achieve our result, we develop a lower bound technique based on a geometric quantity which we dub approximation radius. Using Fano's inequality and the refinement developed in [23, 18, we show that the minimax risk of a convex body is lower bounded by that of the ball with radius equal to the approximation radius of that body. Compared to the existing lower bound techniques, such as the Bernstein bound and the bound followed from considering the worst (typically discrete) distributions (see [14, 20] and [4, 6]), the approximation radius relies on a volume estimation and is both convenient to operate and flexible to provide strong lower bounds.

One center piece in this paper is the connection established between the approximation radius and the Kolmogorov width. Towards this step, we use some results developed in Banach space geometry which was initialized in [1] for investigating the invertibility of matrices with large "robust" rank and subsequently developed by [19, 8]. In particular, we show a duality relationship between the Kolmogorov widths of a convex body and its polar dual body. Our result has a similar flavor to the classical duality in [13] but is tighter when the dimension gap is small.

\section{Preliminaries}

\subsection{Notations and definitions}

For a vector $x=\left(x_{1}, \cdots, x_{n}\right)$ and a real number $p \geq 1$, denote by $\|x\|_{p}$ the $\ell_{p}$-norm of $x$, and $\|x\|_{\infty}=\max _{i}\left|x_{i}\right|$. When $p$ is absent, it means $\ell_{2}$ norm. Let $B_{p}^{n}(x, r)$ denote the $n$ dimensional $\ell_{p}$ ball with radius $r$ and center $x$. Whenever the center is at the origin, it is denoted by $B_{p}^{n}(r)$. Also, we drop the superscript $n$, whenever the dimension is clear from the context, and suppress the argument $r$ for $r=1$.

A set $X \subset \mathbb{R}^{n}$ is called centrally symmetric (or simply symmetric) if for any $x \in X$, we 
have $-x \in X$. For a set $K$, the $\left(\ell_{2}\right)$ radius of $X$ is defined as in the following.

$$
\operatorname{rad}(X)=\max _{x \in K}\|x\|
$$

For $p>0$, and $n \geq 1$, the family $\mathcal{F}_{p}^{m, n}$ is defined as

$$
\mathcal{F}_{p}^{m, n}=\left\{X: X=\left\{x:|A x|_{p} \leq 1\right\}, \text { for } A \in \mathbb{R}^{m \times n}\right\}
$$

In particular, when $p=\infty, \mathcal{F}_{\infty}^{m, n}$ consists of symmetric convex polytopes defined by $m$ hyperplanes. Throughout we consider bounded convex bodies. Our results easily extend to unbounded convex bodies, but the presentation would be cumbersome by including separate case analysis which does not add any new insight.

\subsection{Minimax risk}

Suppose we are given measurements of an unknown $n$-dimensional vector $x$, according to the model

$$
y=x+w,
$$

where $w \in \mathbb{R}^{n}$ follows the normal distribution, $w \sim \mathrm{N}\left(0, \sigma^{2} \mathrm{I}\right)$, and $x$ lies in $X$, a compact convex set in $\mathbb{R}^{n}$. The goal of the minimax estimation problem is to estimate vector $x$, with small error loss, and to evaluate the estimator under the minimax principle.

For any estimator $M: R^{n} \rightarrow R^{n}$, the maximum mean squared error of $M$ on $(X, \sigma)$ is defined as

$$
R(M, X, \sigma)=\max _{x \in X} \mathbb{E}\|x-M(y)\|^{2},
$$

and the minimax risk of $X$ is

$$
R(X, \sigma)=\min _{M} R(M, X, \sigma) .
$$

Estimators generally can be nonlinear function. We denote by $R_{L}(X, \sigma)$ the minimax risk when $M$ is linear. An alternative to the linear and nonlinear estimators is the truncated series estimator [5]. Truncated series estimator is obtained using projections $M(y)=P y$, with $P^{2}=P$. Throughout this paper, projection always mean orthogonal projection. The minimax risk for truncated estimators is defined as

$$
R_{T}(X, \sigma)=\min _{P} \max _{x \in X}\|x-P y\|^{2}
$$

where the minimum is taken over all the linear projections. Since truncated series estimators are linear, we clearly have

$$
R(X, \sigma) \leq R_{L}(X, \sigma) \leq R_{T}(X, \sigma) .
$$

It turns out that the minimax risk for truncated series estimators is completely characterized by the Kolmogorov $k$-width $d_{k}$ of $X$, defined as [16]

$$
d_{k}(X)=\min _{P_{k}} \max _{x \in X}\left\|x-P_{k} x\right\|,
$$


where the minimum is taken over all $k$-dimensional projections. Then, we have

$$
R_{T}(X, \sigma)=\min _{k} d_{k}(X)^{2}+k \sigma^{2} .
$$

For the mean squared error considered in this paper, there is a more direct equivalent definition of the Kolmogorov $k$-width under $\ell_{2}$ metric.

$$
d_{k}(X)=\min _{P \in \mathcal{P}_{k}} \operatorname{rad}(P(X)),
$$

where $\mathcal{P}_{k}$ denotes all the $k$-codimensional (or $n-k$ dimensional) projections, and $\operatorname{rad}(K)$ denotes the $\ell_{2}$ radius of $K$, defined as $\max _{x \in K}\|x\|_{2}$. Furthermore,

$$
\operatorname{rad}(X)=d_{0}(X) \geq d_{1}(X) \geq \ldots \geq d_{n}(X)=0 .
$$

\subsection{Approximation radius}

We define the notion of approximation radius, a geometric measure of any convex body, which as we shall show, provides a lower bound for the minimax risk of the body.

We use $\operatorname{vol}(X)$ to denote the volume of $X$ and $\mathcal{H}_{n}^{k}$ to denote all the $k$ dimensional subspaces in $\mathbb{R}^{n}$. Assume $X \subseteq \mathbb{R}^{n}$ is a convex body that contains the origin. For any $r>0$, the volume ratio $\operatorname{vr}(X, r)$ of $X$ is defined as

$$
\operatorname{vr}(X, r)=\left(\frac{\operatorname{vol}\left(X \cap B_{2}^{n}(r)\right)}{\operatorname{vol}\left(B_{2}^{n}(r)\right)}\right)^{1 / n},
$$

and the $k$-volume ratio $\operatorname{vr}_{k}(X, r)$ of $X$ is defined as the maximum volume ratio over all the $k$ dimensional central cut of $X$, i.e.

$$
\operatorname{vr}_{k}(X, r)=\max _{H \in \mathcal{H}_{n}^{k}} \operatorname{vr}(X \cap H, r) .
$$

Clearly, $0 \leq \operatorname{vr}(X, r) \leq 1$. Further,

Fact 2.1. If $X$ is convex and contains the origin, then $\operatorname{vr}(X, r)$, and hence $\operatorname{vr}_{k}(X, r)$ for any $k$, is non-increasing in $r$.

Proof. It suffices to show for any $c>1, \operatorname{vr}(X, c \cdot r) \leq \operatorname{vr}(X, r)$.

$$
X \cap B_{2}^{n}(c \cdot r)=c\left(\frac{1}{c} X \cap B_{2}^{n}(r)\right) \subseteq c\left(X \cap B_{2}^{n}(r)\right),
$$

where $\frac{1}{c} X \subseteq X$ follows from the assumption that $X$ is convex and contains the origin. Therefore $\operatorname{vol}\left(X \cap B_{2}^{n}(c \cdot r)\right) \leq c^{n} \operatorname{vol}\left(X \cap B_{2}^{n}(r)\right)$. The claim follows immediately from the definition of volume ratio and the identity $\operatorname{vol}\left(B_{2}^{n}(c \cdot r)\right)=c^{n} \operatorname{vol}\left(B_{2}^{n}(r)\right)$.

Central to lower bounding the minimax risk is the notion of approximation radius.

Definition 2.2. For $0 \leq c \leq 1$, and integer $1 \leq k \leq n$, the $(c, k)$-approximation radius of $X$, denoted by $z_{c, k}(X)$, is defined as the maximum $r$ such that $\operatorname{vr}_{k}(X, r) \geq c$, i.e.

$$
z_{c, k}(X)=\sup \left\{r: \operatorname{vr}_{k}(X, r) \geq c\right\} .
$$

Note that if $X$ contains the origin in its interior, then $z_{c, k}(X)$ is always defined for $0 \leq c \leq 1$ 


\subsection{Polar dual of convex bodies}

The connection between the Kolmogorov width and the approximation radius is established via the polar dual of the body. We state some basic facts about the polar dual body which we will need later.

Definition 2.3. For any $K \subset R^{n}$, denote by $K^{\circ}$ the (polar) dual set of $K$,

$$
K^{\circ}=\{y \mid x \cdot y \leq 1 \quad \text { for all } x \in K\} .
$$

If $K$ lies on a lower dimensional subspace, $K^{\circ}$ is understood as the dual set on the lowest dimensional subspace that contains $K$.

Fact 2.4. If $X=\left\{x:|A x|_{\infty} \leq 1\right\}$, then $X^{\circ}=A^{T} B_{1}^{m}$.

Fact 2.5. Let $H$ be a subspace of $R^{n}$. Denote by $P_{H}$ the projection on $H$. Then $P_{H}\left(K^{\circ}\right)=$ $(H \cap K)^{\circ}$.

Proof. We include a proof of this fact for the sake of completeness. We prove the different but equivalent identity $P_{H}(K)^{\circ}=H \cap K^{\circ}$. Let $m=\operatorname{dim}(H)$ and $H=\left(h_{1}, \ldots, h_{m}\right)$ be an orthonormal basis of $H$. With a slight abuse of notation, we denote by $H$ the matrix in $\mathbb{R}^{n \times m}$ that has $h_{i}$ as columns. Then $P_{H}(x)=H H^{T} x$. Observe that for any $x \in R^{n}, y \in R^{m}$, $\left(H H^{T} x\right) \cdot(H y)=x^{T} H H^{T} H y=x^{T} H y=x \cdot(H y)$. Hence

$$
\begin{aligned}
H y \in P_{H}(K)^{\circ} & \Leftrightarrow \forall x \in K,\left(H H^{T} x\right) \cdot(H y) \leq 1 \\
& \Leftrightarrow \forall x \in K, x \cdot H y \leq 1 \\
& \Leftrightarrow H y \in H \cap K^{\circ} .
\end{aligned}
$$

\section{Main results}

In this paper, we are interested in the minimax risk of the truncated series estimator for symmetric convex bodies. Define $\beta(X)=\max _{\sigma>0} R_{T}(X, \sigma) / R(X, \sigma)$, and $\beta_{p}^{m, n}=\max _{X \in \mathcal{F}_{p}^{m, n}} \beta(X)$. Our main result is

Theorem 3.1. If $n=\Omega(\log m)$, then $\beta_{\infty}^{m, n} \leq c \cdot \log m$, where $c<2 \cdot 10^{8}$. Furthermore, $\beta_{\infty}^{m, n}=\Omega(\sqrt{\log m / \log \log m})$.

The lower bound follows immediately from previous works. As shown in [4] (Theorem $3)$, for the unit $\ell_{1}$ ball $X=B_{1}^{n}, R_{T}(X, 1 / \sqrt{n})=\Omega(1)$ but $R(X, 1 / \sqrt{n})=O(\sqrt{\log n / n})$. Since $B_{1}^{n} \in F_{\infty}^{m, n}$ where $m=2^{n}$, we have $\beta_{\infty}^{m, n}=\Omega(\sqrt{\log m / \log \log m})$ for $n=\Omega(\log m)$. In this paper, our main result is to provide a nearly matching upper bound of $O(\log m)$. The upper bound is the consequence of the following theorems: Theorem 3.2 lower bounds the minimax risk by the approximation radius; Theorems 3.3 , 3.4 together establish a lower bound on the approximation radius by the Kolmogorov width, which in turn upper bounds the minimax risk of the truncated series estimator. We assign concrete values to constants whenever possible. They are purely for presentation clarity and by no means represent the best possible constants. 
Theorem 3.2. There exists a universal constant $C=2 \cdot 46 \cdot 10^{-4}$ such that for any $0<c_{*} \leq 1$,

$$
R(X, \sigma) \geq C c_{*}^{2} \max _{k} \min \left\{z_{c_{*}, k}(X)^{2}, k \sigma^{2}\right\} .
$$

Theorem 3.3. For any convex centrally symmetric $X \subset R^{n}$ and any $0 \leq k \leq n$ and $0<\epsilon<1$,

$$
d_{k}(X) d_{n-(1-\epsilon) k}\left(X^{\circ}\right) \leq c_{1} \sqrt{\frac{k}{\epsilon}}
$$

where $c_{1}=2 /(\sqrt{2}-1) \leq 5$.

Theorem 3.4. Let $X \in \mathcal{F}_{\infty}^{m, n}$. For any $0<c_{*} \leq 0.2$ and $0<k \leq n$,

$$
z_{c_{*}, k}(X) \geq c_{2} \sqrt{\frac{k}{\ln m}} \cdot \frac{1}{d_{n-k}\left(X^{\circ}\right)},
$$

where

$$
c_{2}=0.4 \sqrt{\ln \left(1 /\left(2 c_{*}\right)\right)} .
$$

The paper is mainly devoted to proving Theorems 3.2, 3.3, and 3.4, which together imply Theorem 3.1. We discuss some consequences of our results as well as some open questions at the end.

\section{Lower bounding the minimax risk}

In this section we prove Theorem 3.2, Our starting point is from the obvious lower bound for the Euclidean ball $B_{2}^{n}(r)$. It is well known that $R\left(B_{2}^{n}(r), \sigma\right)=\Omega\left(\min \left(r^{2}, n \sigma^{2}\right)\right)$. We shall show that this is also true for any subset contained in $B_{2}^{n}(r)$ with "non-negligible" (a fraction of $\Omega\left(c^{n}\right)$ for some constant $\left.c>0\right)$ volume.

The proof is based on the information-theoretic bound established in [23. In this technique, the minimax risk is lower bounded by restricting to a maximal finite set of points $\left\{x_{1}, \cdots, x_{r}\right\}$ in $X$, separated from each other by at least an amount $\epsilon$ in the loss metric. Indeed, $\epsilon$ is the maximum separation distance such that the hypothesis $\left\{x_{1}, \cdots, x_{r}\right\}$ are almost indistinguishable. The Fano inequality is then used to relate this indistinguishability to K-L divergence.

We proceed by defining an $\epsilon$-net and a $\delta$-packing in a set $S$.

Definition 4.1. A set $N_{\epsilon} \subseteq S$ is said to be an $\epsilon$-net for $S$ if for any $x \in S$, there exists a $x_{0} \in N_{\epsilon}$, such that $\left\|x-x_{0}\right\| \leq \epsilon$. In addition, a finite set $M_{\delta} \subseteq S$ is said to be an $\delta$-packing in $S$, if for any $x, x^{\prime} \in M_{\delta}, x \neq x^{\prime}$, we have $\left\|x-x^{\prime}\right\|>\delta$.

Proposition 4.2. For any set $X$, let $N_{\epsilon}(X)$ be any $\epsilon$-net for $X$ and $M_{\delta}(X)$ be a $\delta$-packing in $X$. Then,

$$
R(X, \sigma) \geq\left(\frac{\delta}{2}\right)^{2}\left(1-\frac{\log \left|N_{\epsilon}(X)\right|+\frac{\epsilon^{2}}{2 \sigma^{2}}+1}{\log \left|M_{\delta}(X)\right|}\right)
$$


Proposition 4.2 is a direct application of the bound proved in [23] (Theorem 1). For the reader's convenience, we give the details of its derivation in Appendix A

Note that the strongest lower bound in Eq. (12) is achieved per the smallest $\epsilon$-net and the largest $\delta$-packing of $X$. In the following, we will develop an upper bound on the size of the smallest $\epsilon$-net for $X$ and a lower bound for the size of its largest $\delta$-packing.

Lemma 4.3. For any $X \subseteq \mathbb{R}^{n}, r \geq \operatorname{rad}(X)$ and $\epsilon \leq r$, there exists an $\epsilon$-net for $X$, with size at most $(3 r / \epsilon)^{n}$.

The proof of Lemma 4.3 is deferred to Appendix B.

Lemma 4.4. For any $\delta>0$, there exists a $\delta$-packing $M_{\delta}(X)$ with size at least $\frac{\operatorname{vol}(X)}{\operatorname{vol}\left(B_{2}(\delta)\right)}$.

We refer to Appendix C for the proof of Lemma 4.4 .

We are now in position to prove Theorem 3.2 .

Proof. (Theorem 3.2) For any $k$ and $c_{*}$ consider the $k$-dimensional central cross section $Y$ of $X$ that attains the approximation radius $z_{c_{*}, k}$. Let $r_{k}=\min \left\{z_{c_{*}, k}(X), \sqrt{k} \sigma\right\}$, and $Y_{k}=$ $Y \cap B_{2}\left(r_{k}\right)$. Clearly, $R(X, \sigma) \geq R\left(Y_{k}, \sigma\right)$, since $Y_{k} \subseteq X$. We will lower bound $R\left(Y_{k}, \sigma\right)$ by applying Proposition 4.2 and Lemmas 4.3, 4.4,

Since $\operatorname{rad}\left(Y_{k}\right) \leq r_{k}$, by Lemma 4.3 for any $\epsilon \leq r_{k}$, there exists an $\epsilon$-net of $Y_{k}$, say $N$, with $|N| \leq\left(3 r_{k} / \epsilon\right)^{k}$. On the other hand, by Fact 2.1, $\operatorname{vr}_{k}\left(Y, r_{k}\right) \geq \operatorname{vr}_{k}\left(Y, z_{C_{*}, k}(X)\right)=c_{*}$. Therefore

$$
\operatorname{vol}_{k}\left(Y_{k}\right)=\operatorname{vol}_{k}\left(Y \cap B_{2}\left(r_{k}\right)\right)=\operatorname{vr}_{k}\left(Y, r_{k}\right)^{k} \operatorname{vol}_{k}\left(B_{2}^{k}\left(r_{k}\right)\right) \geq c_{*}^{k} \operatorname{vol}_{k}\left(B_{2}^{k}\left(r_{k}\right)\right) .
$$

Combining it with Lemma 4.4, there exists a $\delta$-packing of $Y_{k}$, say $M$, with $|M| \geq$ $c_{*}^{k} \operatorname{vol}_{k}\left(B_{2}^{k}\left(r_{k}\right)\right) / \operatorname{vol}_{k}\left(B_{2}^{k}(\delta)\right)=\left(c_{*} \cdot r_{k} / \delta\right)^{k}$.

Choose $\delta=\left(c_{*} / a\right) r_{k}$, and $\epsilon=r_{k}$, where $a$ is a constant to be determined. Using the bounds on $|N|$ and $|M|$ in Proposition 4.2, we obtain

$$
R\left(Y_{k}, \sigma\right) \geq \frac{1}{4}\left(\frac{c_{*} r_{k}}{a}\right)^{2}\left(1-\frac{k \log 3+\frac{r_{k}^{2}}{2 \sigma^{2}}+1}{k \log a}\right) \geq \frac{1}{4}\left(\frac{c_{*} r_{k}}{a}\right)^{2}\left(1-\frac{\log 3+\frac{3}{2}}{\log a}\right) .
$$

Maximizing the right hand side over $a>1$, we get $a=12.89$. Plugging in for $a$ in Eq. (13), we obtain $R\left(Y_{k}, \sigma\right) \geq C c_{*}^{2} r_{k}^{2}$, with $C=2.46 \cdot 10^{-4}$. Since $1 \leq k \leq n$ is arbitrary, we have

$$
R(X, \sigma) \geq \max _{k} R\left(Y_{k}, \sigma\right) \geq \max _{k} C c_{*}^{2} r_{k}^{2}=C c_{*}^{2} \max _{k} \min \left\{z_{c_{*}, k}(X)^{2}, k \sigma^{2}\right\} .
$$

Invoking relation (5) and Theorem 3.2, in order to prove the near optimality of truncated series estimators for family $\mathcal{F}_{\infty}^{m, n}$, we establish some properties of the Kolmogorov width and explore its relation to the approximation radius. Before proceeding, we make a comparison between the proposed lower bound, and the one obtained by considering the hardest rectangular sub-problem.

Relation to the hardest rectangular sub-problem. One technique in the literature [5, 14 for lower bounding the minimax risk is to find the "hardest" box contained in the body (or compute the Bernstein width, defined as the side length of the largest cube enclosed in 
the body) and apply the known lower bound for the box. The approximation radius can always be used to achieve at least the same asymptotical lower bound.

Suppose that $X$ contains a box with side lengths $\tau_{1}, \ldots, \tau_{n}$. Then using the box bound [5], we have that $R(X, \sigma)=\Omega\left(\sum_{i} \tau_{i}^{2} \sigma^{2} /\left(\tau_{i}^{2}+\sigma^{2}\right)\right)=\Omega\left(\sum_{i} \min \left(\tau_{i}^{2}, \sigma^{2}\right)\right)$. Now group $\tau_{i}$ 's as follows. The first group consists of $\tau_{1}, \cdots, \tau_{k_{1}}$, where $k_{1}$ is the smallest index such that $\sum_{j=1}^{k_{1}} \min \left(\tau_{j}^{2}, \sigma^{2}\right) \geq \sigma^{2}$. The seconds group consists of $\tau_{k_{1}+1}, \cdots, \tau_{k_{2}}$, where $k_{2}$ is the smallest number such that $\sum_{j=k_{1}+1}^{k_{2}} \min \left(\tau_{j}^{2}, \sigma^{2}\right) \geq \sigma^{2}$, and so forth. Let $k$ be the total number of groups. Firstly, note that $\sum_{i \in I} \min \left(\tau_{i}^{2}, \sigma^{2}\right)$ is at most $2 \sigma^{2}$, for all groups $I$. Hence $k=$ $\Omega\left(\sum_{i} \min \left(\tau_{i}^{2} / \sigma^{2}, 1\right)\right)$. Secondly, by construction, for all groups $I$ (except possibly the last one), we have $\sum_{i \in I} \min \left(\tau_{i}^{2}, \sigma^{2}\right) \geq \sigma^{2}$. Let $k^{\prime}$ be the number of these groups. For each of

them, we can replace the corresponding face by its diagonal with length $\sqrt{\sum_{i \in I} \tau_{i}^{2}} \geq \sigma$. This way we obtain an $k^{\prime}$ dimensional box with each side length at least $\sigma$. Now it is straight forward to see that, $z_{c, k^{\prime}}(X)=\Omega\left(\sqrt{k^{\prime}} \sigma\right)$, and by Theorem 3.2, we get a lower bound of $\Omega\left(k^{\prime} \sigma^{2}\right)=\Omega\left(k \sigma^{2}\right)=\Omega\left(\sum_{i} \min \left(\tau_{i}^{2}, \sigma^{2}\right)\right)$.

\section{A duality relationship for Kolmogorov widths}

We take a detour to establish the connection between the Kolmogorov width and the approximation radius. The connection is via a novel duality relationship between the Kolmogorov widths of $X$ and those of its polar dual, as stated in Theorem 3.3. The proof is an application of some celebrated works in convex geometry [1, 19, 8].

Definition 5.1. A set of vectors $V=\left\{v_{1}, \cdots, v_{s}\right\}$ is called $\delta$-wide if for any $1 \leq i \leq s$, $\operatorname{dist}\left(v_{i}, \operatorname{span}\left[V /\left\{v_{i}\right\}\right]\right) \geq \delta$.

The following proposition concerns an interesting property of $\delta$-wide sets, and can be gleaned from [1, 19, 8]. For reader's convenience, we give the proof of this proposition in Appendix D,

Proposition 5.2. For any $\delta$-wide set $V=\left\{v_{1}, \cdots, v_{s}\right\}$, there exists $\sigma \subseteq\{1, \ldots, s\}$ with $|\sigma| \geq(1-\epsilon)$ s such that for any $\alpha=\left(\alpha_{j}\right)_{j \in \sigma}$,

$$
\left\|\sum_{j \in \sigma} \alpha_{j} v_{j}\right\| \geq c \sqrt{\frac{\epsilon}{s}} \delta \sum_{j \in \sigma}\left|\alpha_{j}\right|,
$$

with $c=(\sqrt{2}-1) / 2$.

Now we use the above proposition to prove Theorem 3.3 .

Proof. (Theorem 3.3) Write $\delta=d_{k}(X)$. Consider the $k+1$ points $V=\left\{v_{1}, \ldots, v_{k+1}\right\}$ inside $X$ which forms the largest $k+1$ simplex. By the maximality of the volume of $V$, for any $1 \leq i \leq k+1$

$$
\operatorname{dist}\left(v_{i}, \operatorname{span}\left[V /\left\{v_{i}\right\}\right]\right)=\max _{x \in X} \operatorname{dist}\left(x, \operatorname{span}\left[V /\left\{v_{i}\right\}\right]\right) .
$$

Note that the vectors in $V$ are affinely independent, and thus $\operatorname{dim}\left(V /\left\{v_{i}\right\}\right)$ is either $k$ or $k-1$. Therefore, there exists an $r$-codimensional projection $P$ such that $\operatorname{Ker}(P)=V /\left\{v_{i}\right\}$, and $r \in\{k-1, k\}$. Then

$$
\operatorname{dist}\left(v_{i}, \operatorname{span}\left[V /\left\{v_{i}\right\}\right]\right)=\left\|P\left(v_{i}\right)\right\| .
$$


Also, by Eq. (14), we have

$$
\|P(x)\|=\operatorname{dist}\left(x, \operatorname{span}\left[V /\left\{v_{i}\right\}\right]\right) \leq \operatorname{dist}\left(v_{i}, \operatorname{span}\left[V /\left\{v_{i}\right\}\right]\right)=\left\|P\left(v_{i}\right)\right\|,
$$

for any $x \in X$. On the other hand, since $d_{r}(X) \geq d_{k}(X)=\delta$ and $X$ is centrally symmetric, there exist $x, y \in X$, such that $\|P(x)-P(y)\| \geq 2 \delta$. Hence

$$
\left\|P\left(v_{i}\right)\right\| \geq \frac{1}{2}(\|P(x)\|+\|P(y)\|) \geq \frac{1}{2}\|P(x)-P(y)\| \geq \delta .
$$

Using Eq. (15), $V$ is $\delta$-wide. By Proposition 5.2, there exists $\sigma \subseteq\{1, \ldots, k\}$ with $|\sigma| \geq(1-\epsilon) k$ such that for any $\alpha=\left(\alpha_{j}\right)_{j \in \sigma}$,

$$
\left\|\sum_{j \in \sigma} \alpha_{j} v_{j}\right\| \geq c \sqrt{\frac{\epsilon}{k}} \delta \sum_{j \in \sigma}\left|\alpha_{j}\right|, \quad c=\frac{\sqrt{2}-1}{2} .
$$

Let $H=\operatorname{span}\left[\left\{v_{i} \mid i \in \sigma\right\}\right]$. We claim that

$$
H \cap X \supseteq H \cap B_{2}^{n}(c \sqrt{\epsilon / k} \delta) .
$$

Consider $Y=\left\{\sum_{j \in \sigma} \alpha_{j} v_{j}\left|\sum_{j \in \sigma}\right| \alpha_{j} \mid \leq 1\right\}$. Since $X$ is convex and centrally symmetric, and $\left\{v_{i}\right\} \subseteq H \cap X$, we have $Y \subseteq H \cap X$. Hence, it suffices to show that $H \cap B_{2}^{n}(c \sqrt{\epsilon / k} \delta) \subseteq Y$. For any given $x \in H \cap B_{2}^{n}(c \sqrt{\epsilon / k} \delta)$, let $r^{*}=\max \{r: r x \in Y\}$. Clearly, there exists $\alpha=(\alpha)_{j \in \sigma}$, such that, $r^{*} x=\sum_{j \in \sigma} \alpha_{j} v_{j}$, and $\sum_{j \in \sigma}\left|\alpha_{j}\right|=1$. Hence,

$$
\left\|r^{*} x\right\|=\left\|\sum_{j \in \sigma} \alpha_{j} v_{j}\right\| \geq c \sqrt{\frac{\epsilon}{k}} \delta \sum_{j \in \sigma}\left|\alpha_{j}\right|=c \sqrt{\frac{\epsilon}{k}} \delta .
$$

As $x \in H \cap B_{2}^{n}(c \sqrt{\epsilon / k} \delta)$, we have $\|x\| \leq c \sqrt{\epsilon / k} \delta$ and by Eq. (18), we obtain $r^{*} \geq 1$. Consequently, $x \in Y$. Since $x \in H \cap B_{2}^{n}(c \sqrt{\epsilon / k} \delta)$ was arbitrary, we have

$$
H \cap B_{2}^{n}(c \sqrt{\epsilon / k} \delta) \subseteq Y \subseteq H \cap X .
$$

By Fact 2.5,

$$
\begin{aligned}
P_{H}\left(X^{\circ}\right) & =(H \cap X)^{\circ} \\
& \subseteq\left(H \cap B_{2}^{n}(c \sqrt{\epsilon / k} \delta)\right)^{\circ} \\
& =H \cap B_{2}\left(\frac{1}{c \sqrt{\epsilon / k} \delta}\right) .
\end{aligned}
$$

$\operatorname{Thus} \operatorname{rad}\left(P_{H}\left(X^{\circ}\right)\right) \leq 1 /(c \sqrt{\epsilon / k} \delta)$. Note that $P_{H} \in \mathcal{P}_{n-\operatorname{dim}(H)}$. Hence,

$$
d_{n-\operatorname{dim}(H)}\left(X^{\circ}\right)=\min _{P \in \mathcal{P}_{n-\operatorname{dim}(H)}} \operatorname{rad}\left(P\left(X^{\circ}\right)\right) \leq \frac{1}{c \sqrt{\epsilon / k} \delta} .
$$

Since $\operatorname{dim}(H)=|\sigma| \geq(1-\epsilon) k$, recalling Eq. (6) $), d_{n-(1-\epsilon) k}\left(X^{\circ}\right) \leq d_{n-\operatorname{dim}(H)}\left(X^{\circ}\right)$. Taking $c_{1}=1 / c=2 /(\sqrt{2}-1)$, we have

$$
d_{k}(X) d_{n-(1-\epsilon) k}\left(X^{\circ}\right) \leq c_{1} \sqrt{\frac{k}{\epsilon}} .
$$


Before we pass to the next section, we make a few remarks about the duality relationship stated in Theorem 3.3 .

Remark 5.3. The dependence on $k$ is the best possible. Consider $X=B_{1}^{n}$, the unit $\ell_{1}$ ball. Then $X^{\circ}=B_{\infty}^{n}$. It is easy to see that for any $0 \leq k, k^{\prime} \leq n, d_{k}(X) \geq \sqrt{1-k / n}$ and $d_{n-k^{\prime}}\left(X^{\circ}\right) \geq \sqrt{k^{\prime}}$. When $k \leq n / 2$ and $k^{\prime}=\Omega(k)$, we have that $d_{k}(X) d_{n-k^{\prime}}\left(X^{\circ}\right)=\Omega(\sqrt{k})$. We do not know if the dependence on $\epsilon$ is the best possible. But for the application in this paper, the dependence on $\epsilon$ is not significant as it will be chosen as a constant.

Remark 5.4. By using the maximum volume ellipsoid, it is fairly easy to show that for any $0 \leq k<n$,

$$
d_{k}(X) d_{n-k-1}\left(X^{\circ}\right) \leq \sqrt{n} .
$$

Consider the maximal enclosed ellipsoid $E \subseteq X$. By John's theorem [10], $E \subseteq X \subseteq \sqrt{n} E$. Let the axes lengths of $E$ be $\lambda_{1} \geq \lambda_{2} \ldots \geq \lambda_{n} \geq 0$. Then $d_{k}(X) \leq \sqrt{n} \lambda_{k+1}$ since $X \subseteq \sqrt{n} E$. On the other hand, by duality $X^{\circ} \subseteq E^{\circ}$. The axes lengths of $E^{\circ}$ are $1 / \lambda_{n} \geq \ldots \geq 1 / \lambda_{2} \geq$ $1 / \lambda_{1}$. So $d_{n-k-1}\left(X^{\circ}\right) \leq 1 / \lambda_{k+1}$. Therefore, $d_{k}(X) d_{n-k-1}\left(X^{\circ}\right) \leq \sqrt{n}$.

However, proving the stated bound requires more advanced tool (Proposition 5.2) .

Remark 5.5. In [13], a duality about Gelfand numbers are given, where Gelfand number $c_{k}$ is defined as

$$
c_{k}(X)=\min _{H: \operatorname{codim}(H)=k} \operatorname{rad}(H \cap X) .
$$

Observe that $c_{k} \leq d_{k}$. To put it in a comparable form, in [13], it is shown that there exists constant $D>0$, such that for any $0<\kappa<1, c_{k}(X) c_{(1-\kappa) n-k-D}\left(X^{\circ}\right)=O(1 / \kappa)$. This duality relation focuses on the duality gap, i.e. the product can be upper bounded by any constant. In our case there is a factor of $\sqrt{k}$. However, the dimension gap, i.e. the difference between the dimension in one term and the co-dimension in the other term, is $\kappa n$ in this relationship. But ours is $\epsilon k$, much smaller when $k$ is small. If we were to apply the duality in [13], we then need to set $\kappa=\epsilon k / n$, resulting in a bound of $O(n /(\epsilon k))$, much larger than our bound when $k$ is small. But the duality in [13] holds with high probability for a randomly chosen subspace, while it is not true for our bound.

\section{Main theorem}

In the previous section, we showed a relationship between the Kolmogorov widths of $X$ and its polar dual $X^{\circ}$. This easily translates to a relation between the Kolmogorov width and the radius of the largest Euclidean ball contained in $X$, which in turn gives us a bound on the minimax risk of the truncated series estimator. However, the bound is fairly weak due to the large duality gap of $\sqrt{k}$. If it were some constant in place of $\sqrt{k}$, we would already obtain the results we search after. Unfortunately per Remark [5.3, this dependence cannot be improved. In this section, we show that if $X$ is defined by $m$ hyperplanes, we can scale the largest Euclidean ball contained in $X$ by the factor of $\sqrt{k / \log m}$ such that the fraction of its volume inside $X$ is still non-negligible, despite that the scaled ball may grow outside of $X$. This gives us the proof of Theorem 3.4, and therefore of Theorem 3.1. 
Proof. (Theorem 3.4) Let $X$ be an arbitrary element in $\mathcal{F}_{\infty}^{m, n}$. Hence, there exists $A \in \mathbb{R}^{m \times n}$ such that $X=\left\{x \in \mathbb{R}^{n}:|A x|_{\infty} \leq 1\right\}$. Let $\tau=d_{n-k}\left(X^{\circ}\right)$. By definition of Kolmogorov widths, there exists a subspace $H$ with $\operatorname{dim}(H)=k$, such that $\operatorname{rad}\left(P_{H}\left(X^{\circ}\right)\right)=\tau$. Let $H=\left(h_{1}, \ldots, h_{k}\right)$, where $h_{i}$ 's are any orthonormal bases of $H$. By Fact 2.4, $X^{\circ}=A^{T} B_{1}^{m}$, and $P_{H}\left(X^{\circ}\right)=H H^{T} A^{T} B_{1}^{m}$. As $\operatorname{rad}\left(P_{H}\left(X^{\circ}\right)\right)=\tau$, for any $y \in P_{H}\left(X^{\circ}\right),\|y\| \leq \tau$. Equivalently, for any $w \in B_{1}^{m},\left\|H^{T} A^{T} w\right\| \leq \tau$. Let $F=A H$ and write $F=\left(f_{i j}\right)_{m \times k}$. By duality of matrix norms,

$$
\max _{w \in B_{1}}\left\|F^{T} w\right\|=\max _{1 \leq i \leq m} \sqrt{\sum_{j=1}^{k} f_{i j}^{2}} .
$$

Since $\left\|F^{T} w\right\| \leq \tau$ for $w \in B_{1}^{m}$, we have $\sqrt{\sum_{j} f_{i j}^{2}} \leq \tau$, for any $1 \leq i \leq m$.

Consider a random vector $g=\left(g_{1}, \ldots, g_{k}\right)$ where $g_{i}$ 's are i.i.d. standard gaussians. Denote by $\mu$ the probability density function of $g$, i.e.,

$$
\mu(g)=\frac{1}{(2 \pi)^{k / 2}} \exp \left\{-\frac{1}{2} \sum_{i=1}^{k} g_{i}^{2}\right\} .
$$

Let $\mu_{0}=1 /(2 \pi)^{k / 2}, r=\sqrt{2 k \ln \left(1 /\left(2 c_{*}\right)\right)}$, and $\mu_{1}=\mathbb{P}(\|g\| \leq r)$.

Using the standard tail bound for sum of random normal variables [7], for any constant $c>0$, and for any $1 \leq i \leq m$,

$$
\mathbb{P}\left\{\left|(F g)_{i}\right| \geq c \sqrt{\left(\sum_{j=1}^{k} f_{i j}^{2}\right) \ln m}\right\} \leq m^{-c^{2} / 2} .
$$

Since $\sqrt{\sum_{j} f_{i j}^{2}} \leq \tau$, we obtain

$$
\mathbb{P}\left\{\left|(F g)_{i}\right| \geq c \tau \sqrt{\ln m}\right\} \leq m^{-c^{2} / 2} .
$$

Applying union bound for $1 \leq i \leq m$, we obtain

$$
\mathbb{P}\left\{F g \in c \tau \sqrt{\ln m} B_{\infty}^{m}\right\}=1-\mathbb{P}\left(\cup_{i=1}^{m}\left\{\left|(F g)_{i}\right| \geq c \tau \sqrt{\ln m}\right\}\right) \geq 1-m^{1-c^{2} / 2} .
$$

Consequently,

$$
\begin{aligned}
\mathbb{P}\{H g \in c \tau \sqrt{\ln m} X\} & =\mathbb{P}\left\{|A H g|_{\infty} \leq c \tau \sqrt{\ln m}\right\} \\
& =\mathbb{P}\left\{|F g|_{\infty} \leq c \tau \sqrt{\ln m}\right\} \geq 1-m^{1-c^{2} / 2} .
\end{aligned}
$$

Assuming $m \geq 2$, and letting $c=\sqrt{4-2 \log _{2} \mu_{1}}$, we obtain $\mathbb{P}\{H g \in c \tau \sqrt{\ln m} X\} \geq$ $1-\mu_{1} / 2$. Note that the function $\mu(g)$ is decreasing in $\|g\|$. Therefore,

$$
\begin{aligned}
\operatorname{vol}\left(c \tau \sqrt{\ln m} X \cap B_{2}^{k}(r)\right) & \geq \frac{1}{\mu_{0}} \mathbb{P}\left\{H g \in\left(c \tau \sqrt{\ln m} X \cap B_{2}^{k}(r)\right)\right\} \\
& \geq \frac{1}{\mu_{0}}\left(\mathbb{P}(H g \in c \tau \sqrt{\ln m} X)+\mathbb{P}\left(H g \in B_{2}^{k}(r)\right)-1\right) \\
& \geq \frac{1}{\mu_{0}}\left(1-\frac{\mu_{1}}{2}+\mu_{1}-1\right)=\frac{\mu_{1}}{2 \mu_{0}} .
\end{aligned}
$$

Here, $B_{2}^{k}(r)$ is the $k$ dimensional $\ell_{2}$ ball in the subspace $H$. (Recall that $\operatorname{dim}(H)=k$ ). 
Fact 6.1. Let $\mu_{0}=1 /(2 \pi)^{k / 2}, r=\sqrt{2 k \ln \left(1 /\left(2 c_{*}\right)\right)}$, and $\mu_{1}=\mathbb{P}(\|g\| \leq r)$. The followings hold true.

(a) $\mu_{1} \geq \mu_{0} e^{-r^{2} / 2} \operatorname{vol}\left(B_{2}^{k}(r)\right)$.

(b) If $0<c_{*} \leq 0.2$, then

$$
\mu_{1} \geq 1-2 c_{*} \sqrt{2 e \ln \frac{1}{2 c_{*}}} \geq 0.1 .
$$

We refer to Appendix E for the proof of Fact 6.1.

Using Fact 6.1 (part $(a)$ ) in Eq. (26), we get

$$
\left\{\frac{\operatorname{vol}\left(c \tau \sqrt{\ln m} X \cap B_{2}^{k}(r)\right)}{\operatorname{vol}\left(B_{2}^{k}(r)\right)}\right\}^{\frac{1}{k}} \geq \frac{1}{2^{1 / k}} e^{-r^{2} / 2 k}=\frac{2 c_{*}}{2^{1 / k}} \geq c_{*} .
$$

Scaling the sets by factor $1 /(c \tau \sqrt{\ln m})$ in the left hand side of Eq. (28) $)$, and using the definition of approximation radius,

$$
z_{c_{*}, k}(X) \geq \frac{r}{c \tau \sqrt{\ln m}}=c_{2} \sqrt{\frac{k}{\ln m}} \cdot \frac{1}{d_{n-k}\left(X^{\circ}\right)},
$$

where $c_{2}=(\sqrt{2} / c) \sqrt{\ln \left(1 /\left(2 c_{*}\right)\right)}$. Using Fact 6.1 (part $\left.(b)\right)$,

$$
c=\sqrt{4-2 \log _{2} \mu_{1}} \leq 3.3,
$$

whence we obtain $c_{2} \geq 0.4 \sqrt{\ln \left(1 /\left(2 c_{*}\right)\right)}$. This concludes the proof.

With all these preparations, we can now prove the main theorem.

Proof. (Theorem 3.1) As mentioned earlier, the lowerbound is implied by previous work. We only show the upperbound. Recall that $d_{k}(X)$ is non-decreasing in $k$. (see Eq. (6)). Let $k^{*}=\min \left\{k \geq 1 \mid d_{k}(X)^{2} \leq k \sigma^{2}\right\} . \quad\left(k^{*}\right.$ exists since $\left.d_{n}(X)=0\right)$. Consider the two cases below separately.

- $\left(\mathbf{k}^{*}>\mathbf{1}\right)$ : Invoking Eq. (5),$R_{T}(X, \sigma) \leq d_{k^{*}}(X)^{2}+k^{*} \sigma^{2}$. By definition of $k^{*}, R_{T}(X, \sigma) \leq$ $2 k^{*} \sigma^{2}$. Further,

$$
\begin{aligned}
d_{k^{*}}(X)^{2}+k^{*} \sigma^{2} & \leq d_{k^{*}-1}(X)^{2}+\frac{k^{*}}{k^{*}-1}\left(k^{*}-1\right) \sigma^{2} \\
& \leq d_{k^{*}-1}(X)^{2}+2 d_{k^{*}-1}(X)^{2}=3 d_{k^{*}-1}(X)^{2}
\end{aligned}
$$

Hence, $R_{T}(X, \sigma) \leq 3 \min \left\{d_{k^{*}-1}(X)^{2}, k^{*} \sigma^{2}\right\}$. On the other hand,

$$
\begin{aligned}
z_{c_{*},\left(k^{*}-1\right) / 2}(X) & \geq c_{2} \sqrt{\frac{k^{*}-1}{2 \ln m}} \cdot \frac{1}{d_{n-\left(k^{*}-1\right) / 2}\left(X^{\circ}\right)} \\
& \geq \frac{c_{2}}{2 c_{1} \sqrt{\ln m}} d_{k^{*}-1}(X),
\end{aligned}
$$


where the first inequality follows from Theorem 3.4 and the second one follows from Theorem 3.3. Applying Theorem 3.2 ,

$$
\begin{aligned}
R(X, \sigma) & \geq C c_{*}^{2} \min \left\{z_{c_{*},\left(k^{*}-1\right) / 2}(X)^{2}, \frac{k^{*}-1}{2} \sigma^{2}\right\} \\
& \geq C c_{*}^{2} \min \left\{\frac{c_{2}^{2}}{4 c_{1}^{2} \ln m} d_{k^{*}-1}(X)^{2}, \frac{k^{*}-1}{2} \sigma^{2}\right\} \\
& \geq \frac{C c_{*}^{2}}{4 \ln m} \min \left(c_{2}^{2} / c_{1}^{2}, 1\right) \min \left\{d_{k^{*}-1}(X)^{2}, k^{*} \sigma^{2}\right\} \\
& \geq \frac{C_{1}}{\ln m} R_{T}(X, \sigma),
\end{aligned}
$$

for $C_{1}=\left(C c_{*}^{2} / 12\right) \min \left(c_{2}^{2} / c_{1}^{2}, 1\right)$.

- $\left(\mathbf{k}^{*}=\mathbf{1}\right)$ : Using Eq. (15),

$$
R_{T}(X, \sigma) \leq \min \left\{d_{0}(X)^{2}, d_{1}(X)^{2}+\sigma^{2}\right\} \leq \min \left\{\operatorname{rad}(X)^{2}, 2 \sigma^{2}\right\},
$$

where we used the assumption $k^{*}=1$ in the final step. On the other hand, $X$ contains a segment $S$ with length $\operatorname{rad}(X)$. Using the result of [5],

$$
R(X, \sigma) \geq R(S, \sigma)=\frac{\sigma^{2} \cdot \operatorname{rad}(X)^{2}}{\sigma^{2}+\operatorname{rad}(X)^{2}} \geq \frac{1}{2} \min \left(\sigma^{2}, \operatorname{rad}(X)^{2}\right)
$$

Therefore, $R(X, \sigma) \geq(1 / 4) R_{T}(X, \sigma)$.

Combining both cases, we have

$$
\frac{R_{T}(X, \sigma)}{R(X, \sigma)} \leq M_{c_{*}} \ln m,
$$

where

$M_{c_{*}}=\frac{1}{C_{1}}=\frac{12}{C c_{*}^{2}} \max \left(c_{1}^{2} / c_{2}^{2}, 1\right), C=2.46 \cdot 10^{-4}, c_{1}=2 /(\sqrt{2}-1), c_{2}=0.4 \sqrt{\ln \left(1 /\left(2 c_{*}\right)\right)}$.

Minimizing $M_{c_{*}}$ over $0<c_{*} \leq 0.2$, we obtain $c_{*}=0.2$ with $M_{c_{*}}<2 \cdot 10^{8}$.

Remark 6.2. It is essential that $X$ is symmetric. Otherwise, we can take an orthant of $B_{1}^{n}$ which has $O(n)$ faces and has large gap between $R_{T}(X, \sigma)$ and $R(X, \sigma)$.

\section{Discussions}

\subsection{Applications to estimating Lipschitz functions}

The problem of estimating values of a Lipschitz function, at a set of sampled points, from noisy measurements is discussed in the introduction. Since the Lipschitz condition can be represented as linear conditions, Theorem 3.1 is widely applicable to such problems. For example, the function can be defined on any metric space, the sampling points can be arbitrary set of points, and the Lipschitz condition can be of higher order. As long as the corresponding linear constraints is bounded by $n^{O(1)}$ for $n$ samples, the approximation factor is within a small factor of $O(\log n)$ of the optimal. 


\subsection{Smooth convex bodies}

In the above, we have shown that $\beta_{\infty}^{m, n}=O(\log m)$. The celebrated Pinsker bound [17] states that $\beta_{2}^{m, n}=O(1)$. What about $\beta_{p}^{m, n}$ for other $p$ 's? By plugging $\sigma=1 / \sqrt{n}$ in Theorem 3 in [4], we have that for $1 \leq p<2, \beta_{p}^{n, n}=\Omega\left((n / \log n)^{1-p / 2}\right)$. So we will not be able to obtain a similar bound to Theorem 3.1 when $p<2$. On the other hand, we conjecture that similar upperbound holds when $p \geq 2$.

Conjecture 7.1. For any $p \geq 2$, there exists a constant $C=C(p)$, such that for any $m, n \geq 2, \beta_{p}^{m, n} \leq C \log m$.

Define the distance $d(X, Y)$ between two centrally symmetric convex body $X, Y$ as the smallest $c$ such that there exists a uniformly scaled orthogonal transformation $F$ such that $F Y \subseteq X \subseteq c F Y$. We note that $d(\cdot, \cdot)$ is similar to but different from the classical BanachMazur distance in which $F$ is any linear transformation. and that $\log d(\cdot, \cdot)$ is a pseudometric (non-negative, symmetric, and with triangular inequality). By straightforward arguments, $\beta(X) \leq d(X, Y)^{2} \beta(Y)$. Since $d\left(B_{p}^{n}, B_{2}^{n}\right)=n^{1 / 2-1 / p}$ and $d\left(B_{p}^{n}, B_{\infty}^{n}\right)=n^{1 / p}$, we have the following nontrivial bound.

Corollary 7.2. For $p \geq 2, \beta_{p}^{m, n}=O\left(\min \left(n^{1-2 / p}, m^{2 / p} \log m\right)\right)$. In particular, for $p \geq 2$, $\beta_{p}^{n, n}=O(\sqrt{n \log n})$.

\subsection{Tightness of the approximation radius bound}

We have used the approximation radius to lower bound the minimax risk of a convex body $X$. How tight is this bound? This paper has shown that it is at least within $O(\log m)$ factor of the optimal upper bound, and it is achieved by using the (rather limited) truncated series estimators.

As discussed before, the approximation radius provides a lower bound at least as good as using Bernstein width, which is known to be asymptotically optimal for $B_{p}^{n}$ when $p \geq 2$. In this section, we consider $B_{p}^{n}$ for $1 \leq p<2$ and show that the lower bound of using approximation radius is very close to the minimax upper bound but does leave a small gap of factor of $\Theta\left((\log n)^{1-p / 2}\right)$.

We start by upper bounding $z_{c, k}(X)$. For any linear $k$-dimensional subspace $H_{k}$, and $B_{2}^{k}(r) \subset H_{k}$, we have

$$
B_{2}^{k}(r) \cap B_{p}^{n} \subseteq H_{k} \cap B_{p}^{n} .
$$

As it is proved in [12], if $1 \leq p \leq 2$, then $\operatorname{vol}\left(H_{k} \cap B_{p}^{n}\right) \leq \operatorname{vol}\left(B_{p}^{k}\right)$. Using the formula for the volume of $k$-dimensional $\ell_{p}$ ball [22], we have

$$
\operatorname{vol}\left(B_{p}^{k}\right)=2^{k} \frac{\Gamma^{k}\left(1+\frac{1}{p}\right)}{\Gamma\left(\frac{k}{p}+1\right)}=\left(\frac{C_{p}}{k^{1 / p}}\right)^{k},
$$

where $C_{p}$ is a constant that depends on $p$. Hence, for any $H_{k}$,

$$
\left(\frac{\operatorname{vol}\left(B_{2}^{k}(r) \cap B_{p}^{n}\right)}{\operatorname{vol}\left(B_{2}^{k}(r)\right)}\right)^{1 / k} \leq\left(\frac{C_{p}^{k}}{C_{2}^{k}} \cdot \frac{k^{k / 2}}{k^{k / p} r^{k}}\right)^{1 / k}=\frac{C_{p}}{C_{2}} \frac{k^{1 / 2-1 / p}}{r} .
$$


Therefore, $z_{c, k}\left(B_{p}^{n}\right) \leq \frac{C_{p}}{C_{2} \cdot c} k^{1 / 2-1 / p}$. For the lower bound of $z_{c, k}\left(B_{p}^{n}\right)$, choose $H_{k}$ to be one of the $k$-dimensional principal subspaces. Then $B_{p}^{n} \cap H_{k}=B_{p}^{k} \supset k^{1 / 2-1 / p} B_{2}^{k}$. Hence, $z_{c, k}\left(B_{p}^{n}\right) \geq \frac{1}{c} k^{1 / 2-1 / p}$. So, $z_{c, k}=\Theta\left(k^{1 / 2-1 / p} / c\right)$. Apply the lower bound in Theorem 3.2 and we obtain $R\left(B_{p}^{n}, \sigma\right)=\Omega\left(\max _{k} \min \left(k^{1-2 / p}, k \sigma^{2}\right)\right)$. When $\sigma \leq 1$, we choose $k \approx \sigma^{-p}$ and obtain a lower bound of $R\left(B_{p}^{n}, \sigma\right)=\Omega\left(\sigma^{2-p}\right)$. By [4], the optimal upper bound for $B_{p}^{n}$ is $R=\Theta\left(\sigma^{2-p}\left(2 \log n \sigma^{p}\right)^{1-p / 2}\right)$ for $(1 / n)^{1 / p} \ll \sigma \ll \sqrt{1 / \log n}$. Hence the approximation radius bound leaves a gap of $\Theta\left((\log n)^{1-p / 2}\right)$. Actually, the largest gap we know of is $\sqrt{\log n}$ by setting $p=1$ in the above bound.

\subsection{Computational complexity}

We have shown that the truncated series estimator is close to optimal for symmetric convex polytopes. For the family of ellipsoids $\mathcal{F}_{2}^{m, n}$, the optimal truncated series estimator can be computed by using the singular value decomposition. However, computing the best truncated series estimator, or the Kolmogorov width, for symmetric convex polytopes, is a hard problem. When $k=0, d_{0}(X)$ is the diameter of $X$, and it is exactly the $\ell_{2}$-norm maximization problem considered in [2]. The problem is NP-hard. Further, it is shown in [2] that it is hard to approximate within any constant factor unless $\mathrm{P}=\mathrm{NP}$.

On the other hand, by using semi-definite programming (SDP) relaxation, one can compute $O(\sqrt{\log m})$ approximation of the diameter [9, 15], i.e. $d_{0}(X)$. However, it is not known how to approximate $d_{k}(X)$ for $k>1$. [21] showed that if the number of vertices of $X$ is $v$, then SDP gives an $O(\sqrt{\log v})$ approximation for $d_{k}$. However, in our problem, the number of vertices of a symmetric convex body could be exponential in $n$. So the technique in [21] does not directly apply to our problem.

\section{Conclusion}

In this paper, we show that the truncated series estimator can achieve nearly optimal minimax risk for symmetric convex bodies defined by few hyperplanes. There are some outstanding open questions raised by this work.

1. What is the best bound for $\beta_{\infty}^{m, n}$ ? Our work leaves a gap of $\Omega(\sqrt{\log m / \log \log m})$ and $O(\log m)$.

2. What is the best bound for $\beta_{p}^{m, n}$ for $p \geq 2$ ? We conjecture it is $O(\log m)$.

3. How tight is the approximation radius bound for lower bounding the minimax risk for convex bodies? For $\ell_{1}$ ball, it has a gap of $\Theta(\sqrt{\log n})$. This is the largest gap we know of.

4. How to efficiently approximate the optimal truncated series estimator for any symmetric convex polytope?

\section{A Proof of Proposition 4.2}

Consider any $M_{\delta}(X)$ - packing in $X$. Let $M_{\delta}(X)=\left\{x_{1}, \cdots, x_{r}\right\}$, and let $u$ be a random variable uniformly distributed on the hypothesis set $\left\{x_{1}, \cdots, x_{r}\right\}$. Denote by $M(y)$, the 
estimation of $x$ given the observation $y$. Define $w=\operatorname{argmin}_{1 \leq j \leq n}\left\|M(y)-x_{j}\right\|$. Since $\| x_{j}-$ $x_{j}^{\prime} \| \geq \delta$, we have $w=j$, if $\left\|M(y)-x_{j}\right\| \leq \delta / 2$. Therefore,

$$
\begin{aligned}
\max _{1 \leq j \leq n} \mathbb{E}_{p_{x_{j}}}\left\|M(y)-x_{j}\right\|^{2} & \geq\left(\frac{\delta}{2}\right)^{2} \max _{1 \leq j \leq n} \mathbb{P}\left\{\left\|M(y)-x_{j}\right\| \geq \frac{\delta}{2} \mid u=j\right\} \\
& \geq \frac{\delta^{2}}{4 r} \sum_{j=1}^{r} \mathbb{P}(w \neq j \mid u=j) \\
& \geq\left(\frac{\delta}{2}\right)^{2} \mathbb{P}(w \neq u) .
\end{aligned}
$$

Let $h(p)$ be the entropy function defined as

$$
h(p)=-p \log p-(1-p) \log (1-p), \quad \text { for } 0 \leq p \leq 1 .
$$

Denote by $H(u \mid w)$ the posterior entropy of $u$, given $w$, and denote by $I(u ; w)$ the mutual information between $u$ and $w$ defined as

$$
I(u ; w)=H(u)-H(u \mid w)=\log r-H(u \mid w) .
$$

Using Fano's inequality ([3], p. 39),

$$
\begin{aligned}
\mathbb{P}(w \neq u) \log (r-1) & \geq H(u \mid w)-h(1 / 2) \\
& =H(u)-I(u ; w)-\log 2 \\
& \geq \log r-I(u ; w)-\log 2
\end{aligned}
$$

We recall the definition of K-L distance between two probability densities $p, q$ on a set $\Omega$, defined as [3],

$$
D_{K L}(p, q)=\int p \log \frac{p}{q} d \mu
$$

where $\mu$ is any measure on $\Omega$.

Using a property of mutual information, and its relation to K-L divergence ( [3], p. 30, 33), we have

$$
\begin{aligned}
I(u ; w)=I(u ; M(y)) \leq I(u ; y) & =\mathbb{E}_{u}\left\{D_{K L}(P(y \mid u), P(y))\right\} \\
& \leq \max _{1 \leq j \leq r} D_{K L}\left(P\left(y \mid x_{j}\right), P(y)\right)
\end{aligned}
$$

Let $N_{\epsilon}(X)$ be any $\epsilon$-net for $X$. Considering the uniform prior distribution on $N_{\epsilon}(X)$, we write, $P(y)=1 /\left|N_{\epsilon}(X)\right| \sum_{\tilde{x} \in N_{\epsilon}(X)} P(y \mid \tilde{x})$. Also, by definition of $\epsilon$-net, for any $x_{j}, 1 \leq j \leq r$, there exists $\tilde{x}_{j} \in N_{\epsilon}(X)$, with $\left\|x_{j}-\tilde{x}_{j}\right\| \leq \epsilon$. Hence,

$$
\begin{aligned}
D_{K L}\left(P\left(y \mid x_{j}\right), P(y)\right) & =\mathbb{E}\left\{\log \frac{P\left(y \mid x_{j}\right)}{\frac{1}{\left|N_{\epsilon}(X)\right|} \sum_{\tilde{x} \in N_{\epsilon}(X)} P(y \mid \tilde{x})}\right\} \\
& \leq \mathbb{E}\left\{\log \frac{P\left(y \mid x_{j}\right)}{\frac{1}{\left|N_{\epsilon}(X)\right|} P\left(y \mid \tilde{x}_{j}\right)}\right\} \\
& =\log \left|N_{\epsilon}(X)\right|+D\left(P\left(y \mid x_{j}\right), P\left(y \mid \tilde{x}_{j}\right)\right)
\end{aligned}
$$


Following the model (4),$y \mid x_{j} \sim \mathrm{N}\left(x_{j}, \sigma^{2} \mathrm{I}\right)$, and $y \mid \tilde{x}_{j} \sim \mathrm{N}\left(\tilde{x}_{j}, \sigma^{2} \mathrm{I}\right)$. Using the definition of K-L distance (Eq. (41)), after some simple algebraic manipulations, we have

$$
D\left(P\left(y \mid x_{j}\right), P\left(y \mid \tilde{x}_{j}\right)\right)=\frac{1}{2 \sigma^{2}}\left\|x_{j}-\tilde{x}_{j}\right\|^{2} \leq \frac{\epsilon^{2}}{2 \sigma^{2}} .
$$

Combining Eqs. (42), (433), and (44), we obtain

$$
I(u ; w) \leq \log \left|N_{\epsilon}(X)\right|+\frac{\epsilon^{2}}{2 \sigma^{2}} .
$$

Using Eq. (39), (40), and (45), we obtain the desired result.

\section{B Proof of Lemma 4.3}

Since $r \geq \operatorname{rad}(X), X \subseteq B_{2}(r)$. Hence, any $\epsilon$-net for $B_{2}(r)$ is also an $\epsilon$-net for $X$. We begin by covering $B_{2}(r)$ with a finite family of balls of radius $\epsilon$. Choose the sequence of centers $p_{1}, p_{2}, \cdots$ in such a way that

$$
p_{i+1} \notin \bigcup_{j=1}^{i} B_{2}\left(p_{j}, \epsilon\right) .
$$

When this is no longer possible, the sequence is terminated. Now the set $P=\left\{p_{i}\right\}$ is an $\epsilon$-net for $B_{2}(r)$. Meanwhile, note that the smaller balls $B_{2}\left(p_{i}, \epsilon / 2\right)$ are all disjoint (since no two of the $p_{i}$ are within distance $\epsilon$ of each other). In addition, $B_{2}\left(p_{i}, \epsilon / 2\right) \subseteq B_{2}(r) \oplus B_{2}(\epsilon / 2)$, where $\oplus$ denotes the Minkowski sum. Therefore,

$$
|P| \operatorname{vol}\left(B_{2}(\epsilon / 2)\right)=\sum_{p_{i} \in P} \operatorname{vol}\left(B_{2}\left(p_{i}, \epsilon / 2\right)\right) \leq \operatorname{vol}\left(B_{2}(r) \oplus B_{2}(\epsilon / 2)\right) .
$$

Evidently, $B_{2}(\epsilon / 2) \subseteq 1 / 2 B_{2}(r)$, since $\epsilon \leq r$. Hence, $B_{2}(r) \oplus B_{2}(\epsilon / 2) \subseteq 3 / 2 B_{2}(r)$, and $\operatorname{vol}\left(B_{2}(r) \oplus B_{2}(\epsilon / 2)\right) \leq(3 / 2)^{n} \operatorname{vol}\left(B_{2}(r)\right)$. Using Eq. (46), we obtain

$$
|P| \leq \frac{(3 / 2)^{n} \operatorname{vol}\left(B_{2}(r)\right)}{\operatorname{vol}\left(B_{2}(\epsilon / 2)\right)} \leq\left(\frac{3 r}{\epsilon}\right)^{n} .
$$

\section{Proof of Lemma 4.4}

Let $M_{\delta}(X)$ denote the maximum size $\delta$-packing of $X$. By maximality of $M_{\delta}(X)$, any other point in $X$ is within $\delta$ distance of one of the points in $M_{\delta}(X)$. Hence,

$$
X \subseteq \bigcup_{p \in M_{\delta}(X)} B_{2}(p, \delta)
$$

whence we obtain

$$
\left|M_{\delta}(X)\right| \geq \frac{\operatorname{vol}(X)}{\operatorname{vol}\left(B_{2}(p, \delta)\right)}
$$




\section{Proof of Proposition 5.2}

The proof is based in a crucial way on the following lemma proved in [8].

Lemma D.1. Let $u_{1}, \cdots, u_{s} \in \mathbb{R}^{n},\left\|u_{i}\right\| \leq 1$. Define the set

$$
E=\left\{\left(\delta_{j}\right)_{j=1}^{s}:\left\|\sum_{j=1}^{s} \delta_{j} u_{j}\right\|^{2} \leq 2 s\right\} .
$$

Then, for every $\epsilon \in(0,1)$, there exists $\sigma \subseteq\{1, \cdots, s\}$ with $|\sigma| \geq(1-\epsilon)$ s, such that

$$
P_{\sigma}(E) \supseteq c \sqrt{\epsilon}[-1,1]^{\sigma}, \quad c=\frac{\sqrt{2}-1}{\sqrt{2}},
$$

where the restriction map $P_{\sigma}$ is defined as $P_{\sigma}:\left(\delta_{j}\right)_{j=1}^{s} \rightarrow\left(\delta_{j}\right)_{j \in \sigma}$.

Since the set $V=\left\{v_{1}, \cdots, v_{s}\right\}$ is $\delta$-wide, there exist $y_{1}, \cdots, y_{s} \in \mathbb{R}^{n}$, so that

$$
\left\langle v_{i}, y_{j}\right\rangle=1_{\{i=j\}}, \quad \text { and } \quad\left\|y_{i}\right\| \leq \frac{1}{\delta}, \quad i, j=1, \cdots, s .
$$

Let $u_{i}=\delta y_{i}$. Applying Lemma D.1, there exists a set $\sigma \subseteq\{1, \cdots, s\}$, with $|\sigma| \geq(1-\epsilon) s$, and $P_{\sigma}(E) \supseteq c \sqrt{\epsilon}[-1,1]^{\sigma}$. Hence we can find $\left(\delta_{j}\right)_{j=1}^{s} \in E$, such that, $\delta_{j}=c \sqrt{\epsilon} \operatorname{sign}\left(\alpha_{j}\right)$, for $j \in \sigma$. Then,

$$
\begin{aligned}
\sum_{j \in \sigma}\left|\alpha_{j}\right| & =\left\langle\sum_{j \in \sigma} \alpha_{j} v_{j}, \sum_{i=1}^{s} \operatorname{sign}\left(\alpha_{i}\right) y_{i}\right\rangle \\
& =\frac{1}{c \sqrt{\epsilon}}\left\langle\sum_{j \in \sigma} \alpha_{j} v_{j}, \sum_{i=1}^{s} \delta_{i} y_{i}\right\rangle \\
& \leq \frac{1}{c \sqrt{\epsilon}}\left\|\sum_{j \in \sigma} \alpha_{j} v_{j}\right\| \cdot \frac{1}{\delta}\left\|\sum_{i=1}^{s} \delta_{i} u_{i}\right\| \\
& \leq \frac{1}{c \delta} \sqrt{\frac{2 s}{\epsilon}}\left\|\sum_{j \in \sigma} \alpha_{j} v_{j}\right\|,
\end{aligned}
$$

where the first step follows form Eq. (48). Rearranging the terms in Eq. (49) implies the result.

\section{E Proof of Fact 6.1}

Proof (Part (a)).

$$
\begin{aligned}
\mu_{1} & =\mathbb{P}(\|g\| \leq r)=\frac{1}{(2 \pi)^{s / 2}} \int_{\|x\| \leq r} e^{-x^{2} / 2} d x \\
& \geq \mu_{0} \int_{\|x\| \leq r} e^{-r^{2} / 2} d x=\mu_{0} e^{-r^{2} / 2} \operatorname{vol}\left(B_{2}^{s}(r)\right) .
\end{aligned}
$$


Proof (Part (b)). We will first upper bound $\mathbb{P}(\|g\|>r)$ using a Chernoff Bound.

$$
\mathbb{P}(\|g\|>r)=\mathbb{P}\left(e^{\lambda \sum_{i=1}^{k} g_{i}^{2}}>e^{\lambda r^{2}}\right) \leq \frac{\mathbb{E}\left\{e^{\lambda \sum_{i=1}^{k} g_{i}^{2}}\right\}}{e^{\lambda r^{2}}} .
$$

Since $g_{i}$ are i.i.d. standard normal variables, it is easy to see that

$$
\mathbb{E}\left\{e^{\lambda \sum_{i=1}^{k} g_{i}^{2}}\right\}=\left(\mathbb{E}\left\{e^{\lambda g_{1}^{2}}\right\}\right)^{k}=\left(\frac{1}{\sqrt{1-2 \lambda}}\right)^{k} .
$$

Using Eq. (52) in Eq. (51) and substituting for $r$, we obtain

$$
\mathbb{P}(\|g\|>r) \leq\left(\frac{1}{\sqrt{1-2 \lambda}} e^{-2 \lambda \ln \frac{1}{2 c_{*}}}\right)^{k} .
$$

Minimizing the right hand side over $\lambda$ gives $\lambda=1 / 2\left(1+1 /\left(2 \ln \left(2 c_{*}\right)\right)\right)$. Notice that $\lambda>0$, for $0<c_{*}<0.2$. Substituting for $\lambda$ in Eq. (53) gives

$$
\mathbb{P}(\|g\|>r) \leq\left(2 c_{*} \sqrt{2 e \ln \frac{1}{2 c_{*}}}\right)^{k} \leq 2 c_{*} \sqrt{2 e \ln \frac{1}{2 c_{*}}},
$$

where the last step follows from $c_{*} \leq 0.2$, and $k \geq 1$. Now, $\mu_{1}=1-\mathbb{P}(\|g\|>r)$. The result follows.

\section{References}

[1] J. Bourgain and L. Tzafriri. Invertibility of 'large' submatrices with applications to the geometry of banach spaces and harmonic analysis. Israel Journal of Mathematics, 57(2):137-224, 1987.

[2] A. Brieden. Geometric optimizatoin problems likely not contained in APX. Discrete and Computational Geometry, 28:201-209, 2002.

[3] T. M. Cover and J. A. Thomas. Elements of Information Theory. Wiley-Interscience, New York, NY, USA, 1991.

[4] D. Donoho and I. M. Johnstone. Minimax risk over $\ell_{p}$-balls for $\ell_{q}$-error. Probability Theory and Related Fields, 99(2):277-303, 1994.

[5] D. Donoho, R. Liu, and B. MacGibbon. Minimax risk over hyperrectangles, and implications. The Annals of Staistics, 18(3):1416-1437, 1990.

[6] D. L. Donoho, I. Johnstone, A. Maleki, and A. Montanari. Compressed sensing over $\ell_{p}$-balls: Minimax mean square error. CoRR, abs/1103.1943, 2011.

[7] W. Feller. An Introduction to Probaility Theory and Its Applications, volume 2. John Wiley and Sons, 2nd edition, 1972.

[8] A. Giannopoulous. A note on the Banach-Mazur distance to the cube. Operator Theory, 77:67-73, 1995. 
[9] M. X. Goemans and D. P. Williamson. Improved approximation algorithms for maximum cut and satisfiability problems using semidefinite programming. J. ACM, 42(6):1115$1145,1995$.

[10] F. John. Extremum problems with inequalities as subsidiary conditions. In Studies and Essays, R. Courant anniversary volume, pages 187-204. Interscience, New York, 1948.

[11] I. M. Johnstone. Gaussian estimation: Sequence and wavelet models. available at http://www-stat.stanford.edu/people/faculty/johnstone, 2011.

[12] M. Meyer and A. Pajor. Sections of the unit ball of $l_{p}^{n}$. Journal of Functional Analysis, 80(1):109-123, 1988.

[13] V. Milman. Spectrum of a position of a convex body and linear duality relations. In Isarel Mathematics Conference Proceedings 3, pages 151-162, 1990.

[14] A. Nemirovski. Topics in Non-parametric Statistics. Lecture Notes in Mathematics. Springer, 1998.

[15] A. Nemirovski, C. Roos, and T. Terlaky. On maximization of quadratic form over intersection of ellipsoids with common center. Mathematical Programming, Series A, 86:463-473, 1999.

[16] A. Pinkus. n-Widths in Approximation Theory. Springer-Verlag, 1984.

[17] M. S. Pinsker. Optimal filtering of square integrable signals in Gaussian white noise. Problems of Information Transmission, 16:120-133, 1980.

[18] G. Raskutti, M. J. Wainwright, and B. Yu. Minimax rates of estimation for highdimensional linear regression over $\ell_{q}$-balls. IEEE Transactions on Information Theory, 57(10):6976-6994, 2011.

[19] S. J. Szarek and M. Talagrand. An isomorphic version of the Sauer-Shelah lemma and the Banach-Mazur distance to the cube. In GAFA Seminar 87-88, Lecture Notes in Mathematics, pages 105-112. Springer-Verlag, 1989.

[20] A. B. Tsybakov. Introduction to Nonparametric Estimation. Springer Series in Statistics. Springer, 2009.

[21] K. R. Varadarajan, S. Venkatesh, Y. Ye, and J. Zhang. Approximating the radii of point sets. SIAM J. Comput., 36(6):1764-1776, 2007.

[22] X. Wang. Volumes of Generalized Unit Balls. Mathematics Magazine, 78(5):390-395, 2005.

[23] Y. Yang and A. Barron. Information-theoretic determination of minimax rates of convergece. The Annals of Statistics, 27(5):1564-1599, 1999. 